PROCEEDINGS OF THE

AMERICAN MATHEMATICAL SOCIETY

Volume 137, Number 10, October 2009, Pages 3543-3545

S 0002-9939(09)09895-5

Article electronically published on May 6, 2009

\title{
HAVING CUT-POINTS IS NOT A WHITNEY REVERSIBLE PROPERTY
}

\author{
EIICHI MATSUHASHI
}

(Communicated by Alexander N. Dranishnikov)

\begin{abstract}
We show that the property of having cut-points is not a Whitney reversible property. This answers in the negative a question posed by Illanes and Nadler.
\end{abstract}

\section{INTRODUCTION}

In this note, all spaces are separable metrizable spaces and maps are continuous. We denote the interval $[0,1]$ by $I$. A compact metric space is called a compactum, and continuum means a connected compactum. If $X$ is a continuum, $C(X)$ denotes the space of all subcontinua of $X$ with the topology generated by the Hausdorff metric.

A topological property $P$ is called a Whitney property provided that if a continuum $X$ has property $P$, so does $\mu^{-1}(t)$ for each Whitney map (see p. 105 of [2] $\mu$ for $C(X)$ and for each $t \in[0, \mu(X))$. A topological property $P^{\prime}$ is called a Whitney reversible property provided that whenever $X$ is a continuum such that $\mu^{-1}(t)$ has property $P^{\prime}$ for all Whitney maps $\mu$ for $C(X)$ and all $t \in(0, \mu(X))$, then $X$ has property $P^{\prime}$. These properties have been studied by many authors (see [2]).

A point $p$ in a continuum $X$ is called a cut-point of $X$ provided that $X \backslash\{p\}$ is disconnected. In this paper we prove that the property of having cut-points is not a Whitney reversible property (it is known that the property of having cut-points is not a Whitney property; see Exercise 43.4 of [2]). This answers in the negative question 43.3 of [2] posed by Illanes and Nadler.

\section{MAIN THEOREM}

A map $f: X \rightarrow Y$ between continua is called an atomic map if $f^{-1}(f(A))=A$ for each $A \in C(X)$ such that $f(A)$ is nondegenerate.

A subcontinuum $T$ of a continuum $X$ is terminal if every subcontinuum of $X$ which intersects both $T$ and its complement must contain $T$. It is known that a map $f$ of a continuum $X$ onto a continuum $Y$ is atomic if and only if every fiber of $f$ is a terminal subcontinuum of $X$.

The main aim of this paper is to prove Theorem 2.2. To prove this theorem, we need the next result, proved by Anderson [1].

Received by the editors December 23, 2008, and, in revised form, January 6, 2009

2000 Mathematics Subject Classification. Primary 54B20; Secondary 54F15.

Key words and phrases. Whitney reversible property, cut-point, terminal continuum, atomic map.

(C)2009 American Mathematical Society 3543

Reverts to public domain 28 years from publication 
Lemma 2.1 (see Theorem of [1]). For each continuum $Y$, there exist a continuum $X$ and a monotone open map $f: X \rightarrow Y$ such that $f^{-1}(y)$ is a nondegenerate terminal subcontinuum of $X$ for each $y \in Y$.

Theorem 2.2. There exists a continuum $Z$ such that:

(A) $Z$ does not have a cut-point, and

(B) $\mu^{-1}(s)$ has a cut-point for each Whitney map $\mu: C(Z) \rightarrow[0, \mu(Z)]$ and for each $s \in(0, \mu(Z))$.

Proof. By Lemma 2.1 there exist a continuum X and a monotone open map $f$ : $X \rightarrow I$ such that $f^{-1}(y)$ is a nondegenerate terminal subcontinuum of $X$ for each $y \in I$. Let $Z$ be the quotient space obtained from $X$ by shrinking $f^{-1}(1)$ to the point. Let $p: X \rightarrow Z$ be the natural projection and $q=f \circ p^{-1}: Z \rightarrow I$. Note that $q$ is a monotone open map such that $q^{-1}(1)$ is a one-point set and $q^{-1}(y)$ is a nondegenerate terminal subcontinuum of $Z$ for each $y \in[0,1)$ (hence $q$ is atomic).

We show that $Z$ has the required properties. At first we prove that $Z$ does not have a cut-point. Let $z \in Z$. If $\{z\}=q^{-1}(1)$, then $Z \backslash\{z\}=q^{-1}([0,1))$. Since $q$ is monotone, $q^{-1}([0,1))$ is connected. Hence in this case $z$ is not a cut-point of $Z$. Assume that $z \in q^{-1}(t)$ for some $t \in[0,1)$ and that $z$ is a cut-point of $Z$. Then there exist nonempty open subsets $O, H \subset Z$ such that $Z \backslash\{z\}=O \cup H$ and $O \cap H=\emptyset$. Then $O \cup\{z\}$ and $H \cup\{z\}$ are nondegenerate continua (see Proposition 6.3 of [3]). We may assume that $q^{-1}(t) \cap H \neq \emptyset$. Since $O \cup\{z\}$ is a nondegenerate continuum, there exists $\left\{z_{i}\right\}_{i=1}^{\infty} \subset O$ such that $\lim _{i \rightarrow \infty} z_{i}=z$. For each $i=1,2, \ldots$, let $t_{i}=q\left(z_{i}\right)$. Note that $q^{-1}\left(t_{i}\right) \subset O$ for each $i=1,2, \ldots$. Since $q$ is an open map, $\lim q^{-1}\left(t_{i}\right)=q^{-1}(t)$. Then $q^{-1}(t) \subset O \cup\{z\}$. This is a contradiction because $q^{-1}(t) \cap H \neq \emptyset$. So $Z$ does not have a cut-point.

Next we prove that $\mu^{-1}(s)$ has a cut-point for each Whitney map $\mu: C(Z) \rightarrow$ $[0, \mu(Z)]$ and for each $s \in(0, \mu(Z))$. Take $a, b \in(0,1)$ such that $\mu\left(q^{-1}([a, b])\right)=s$ (this is possible because $q$ is a monotone open map and $q^{-1}(1)$ is a one-point set). Now we show that

$$
\mu^{-1}(s)=\left\{C \in \mu^{-1}(s) \mid C \subset q^{-1}([0, b])\right\} \cup\left\{C \in \mu^{-1}(s) \mid C \subset q^{-1}([a, 1])\right\} .
$$

If not, there exists $D \in \mu^{-1}(s)$ such that $D \cap q^{-1}([0, a)) \neq \emptyset \neq D \cap q^{-1}((b, 1])$. Then $q(D)$ contains $[a, b]$ as a proper subcontinuum of $q(D)$. Since $q$ is atomic, $D=q^{-1}(q(D))$. So $D$ contains $q^{-1}([a, b])$ as a proper subcontinuum of $D$. This is a contradiction because $\left\{D, q^{-1}([a, b])\right\} \subset \mu^{-1}(s)$. Hence (1) holds.

It is easy to see that

$$
\left\{C \in \mu^{-1}(s) \mid C \subset q^{-1}([0, b])\right\} \cap\left\{C \in \mu^{-1}(s) \mid C \subset q^{-1}([a, 1])\right\}=\left\{q^{-1}([a, b])\right\}
$$

and

$$
\left\{C \in \mu^{-1}(s) \mid C \subset q^{-1}([0, b])\right\} \quad \text { and } \quad\left\{C \in \mu^{-1}(s) \mid C \subset q^{-1}([a, 1])\right\}
$$

are nondegenerate subcontinua of $\mu^{-1}(s)$.

By (11), (2) and (3) we see that $q^{-1}([a, b])$ is a cut-point of $\mu^{-1}(s)$.

By this result, we see that the property of having cut-points is not a Whitney reversible property. 


\section{REFERENCES}

1. R. D. Anderson, Atomic decompositions of continua, Duke Math. J. 23 (1956), 507-514. MR:0082668 (18:590c)

2. A. Illanes and S.B. Nadler Jr., Hyperspaces: Fundamentals and Recent Advances, in: Pure Appl. Math. Ser., Vol. 216, Marcel Dekker, New York, 1999. MR.1670250 (99m:54006)

3. S.B. Nadler Jr., Continuum Theory: An Introduction, Marcel Dekker, New York, 1992. MR:1192552 (93m:54002)

Faculty of Engineering, Yokohama National University, Yokohama, 240-8501, Japan

E-mail address: mateii@ynu.ac.jp 